\title{
Osteoblastoma do capitato: Relato de caso
}

\section{Osteoblastoma of the Capitate Bone: Case Report}

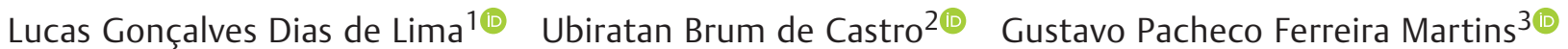

${ }^{1}$ Departamento de Ortopedia e Traumatologia, Hospital das Clínicas, Universidade Federal de Minas Gerais, Belo Horizonte, MG, Brasil

Endereço para correspondência Lucas Gonçalves Dias de Lima, MD,

2 Faculdade de Medicina, Universidade Federal de Minas Gerais, Belo Rua Niquelina, 58, apto. 1.202, Santa Efigênia, Belo Horizonte, MG, Horizonte, MG, Brasil

${ }^{3}$ Hospital das Clínicas, Universidade Federal de Minas Gerais, Belo Horizonte, MG, Brasil

Rev Bras Ortop Brasil (e-mail: lucasgdlima@gmail.com).

\section{Resumo \\ Palavras-chave \\ - ossos do carpo \\ - osteoblastoma \\ - neoplasias ósseas \\ - mão}

Os tumores dos ossos do carpo devem ser investigados em quadros clínicos de dor crônica no punho sem trauma prévio. Gânglio intraósseo, encondroma, osteoma osteoide, e, menos comumente, osteoblastoma são possíveis causas de lesões osteolíticas que afetam os ossos carpais. Na maioria dos casos, a apresentação clínica por si só não é suficiente para diferenciar essas lesões. Conhecer características como o aspecto radiológico e histopatológico de cada uma delas é parte fundamental do diagnóstico diferencial. Apresentamos um caso raro de osteoblastoma do osso capitato, e também fazemos uma revisão da literatura sobre o assunto.

Carpal bone tumors must be investigated in clinical cases of chronic wrist pain with no previous trauma. Intraosseous ganglion, enchondroma, osteoid osteoma, and, less commonly, osteoblastoma are potential causes of osteolytic lesions affecting the carpal bones. In most cases, the clinical presentation alone is not enough to differentiate such lesions. Knowledge of certain characteristics, including the radiological and histopathological aspects of each of these tumors, is critical in order to make the differential diagnosis. We present a rare case of osteoblastoma of the capitate bone and review the literature on the subject.

\section{Introdução}

Os tumores ósseos do carpo devem ser pesquisados diante de um quadro clínico de dor crônica do punho sem histórico de trauma prévio. Quando apresentam características osteolíticas, as causas mais comuns são os gânglios intraósseos, encondroma, osteoma osteóide e, menos comumente, osteoblastoma.

recebido

05 de Outubro de 2020

aceito

28 de Outubro de 2020
DOI https://doi.org/

10.1055/s-0041-1724084. ISSN 0102-3616.
Osteoblastomas são neoplasias benignas formadoras de células ósseas. Representam 1\% dos tumores, e suas localizações mais comuns são os elementos posteriores da coluna vertebral, pelve, e ossos longos. Raramente os ossos do carpo são acometidos, sendo o envolvimento do capitato ainda menos comum. ${ }^{1-3}$ Neste estudo, descreve-se um caso de osteoblastoma do capitato, assim como se faz uma revisão da literatura sobre o tema. (c) 2021. Sociedade Brasileira de Ortopedia e Traumatologia. All rights reserved.

This is an open access article published by Thieme under the terms of the Creative Commons Attribution-NonDerivative-NonCommercial-License, permitting copying and reproduction so long as the original work is given appropriate credit. Contents may not be used for commercial purposes, or adapted, remixed, transformed or built upon. (https://creativecommons.org/ licenses/by-nc-nd/4.0/)

Thieme Revinter Publicações Ltda., Rua do Matoso 170, Rio de Janeiro, RJ, CEP 20270-135, Brazil 


\section{Relato de Caso}

Paciente de 40 anos, destra, com histórico de dor na face dorsal da mão direita, progressiva, com 2 anos de evolução. Negava traumas prévios, infecções, ou outros sintomas associados ao quadro.

Apresentou-se com aumento de volume da área afetada e prejuízo funcional para a realização de atividades básicas de vida diária.

Ao exame, encontrava-se com a flexão do punho direito discretamente limitada, diminuição da força de preensão, e dor à palpação do capitato. A ectoscopia não demonstrava alterações.

As radiografias iniciais mostraram uma lesão osteolítica, bem definida, com margem esclerótica e aspecto lobulado no capitato ( - Figura $\mathbf{1}$ ).

Não foram vistas calcificações ou extensão para partes moles ou outros ossos. Devido ao aspecto inespecífico à radiografia, foi solicitada tomografia computadorizada, que apresentou lesão com componente hipodenso central e margens hiperdensas, bem definidas, restrita à porção distal do capitato (- Figura 2).

Em função do quadro álgico significativo e comprometimento funcional, foi indicado tratamento cirúrgico com ressecção intralesional e preenchimento com enxerto ósseo de olécrano ipsilateral. O material curetado foi enviado para estudo anatomopatológico (-Figura $\mathbf{3}$ ).

A análise dos cortes histológicos dos fragmentos de tecido ósseo mostrou se tratar de lesão benigna composta por tecido ósseo jovem e maduro, sem atipias, permeada por tecido fibroso, sendo diagnosticada como osteoblastoma (- Figura 4).

A avaliação clínica da paciente 1 semana após o procedimento revelou melhora significativa do quadro álgico, o que se manteve nas avaliações posteriores com 3, 6 e 12 semanas de pós-operatório (-Figuras $\mathbf{5}$ ).

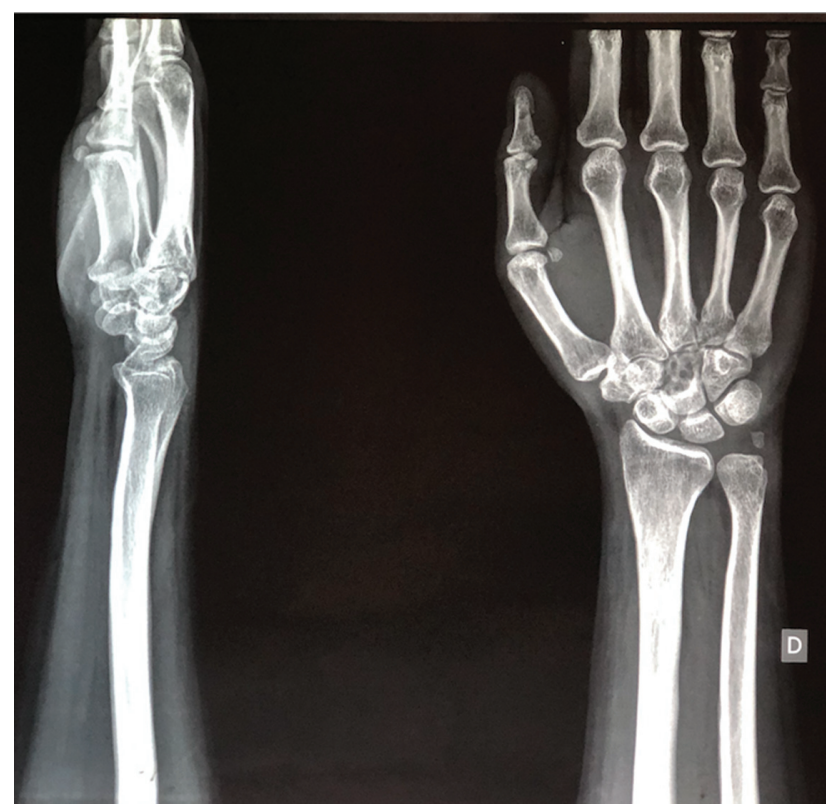

Fig. 1 Radiografia inicial do punho direito. Observa-se presença de lesão osteolítica bem definida no osso capitato.
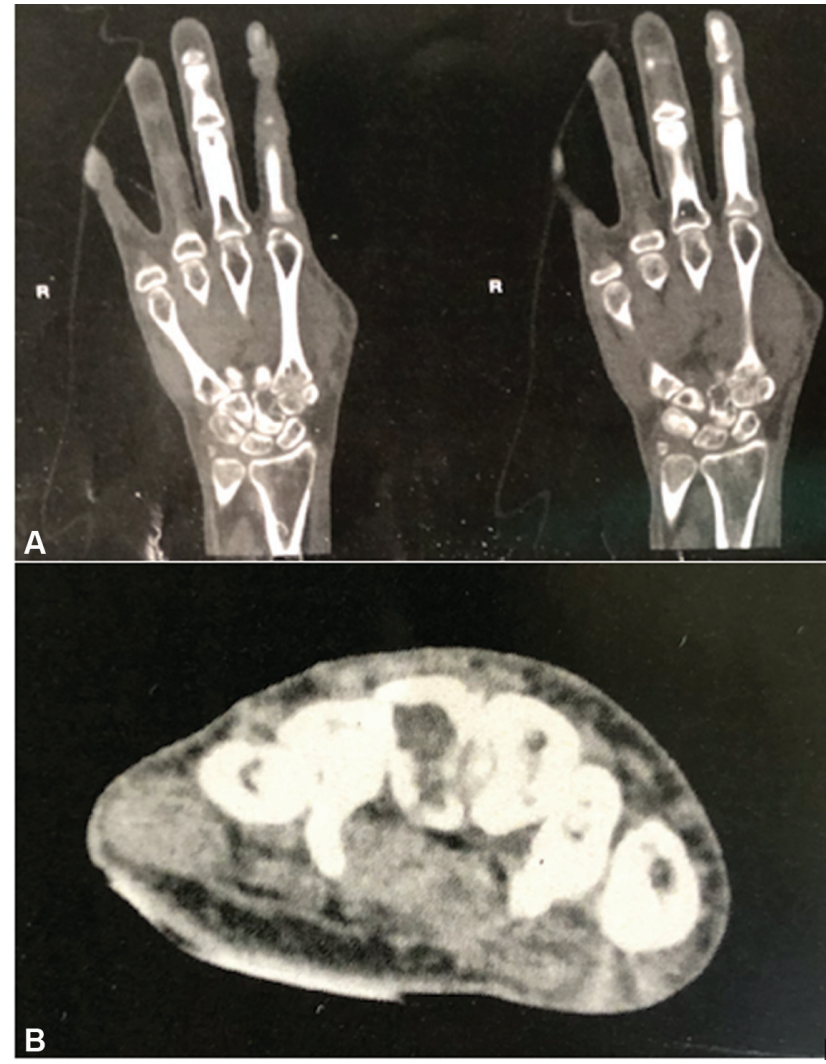

Fig. 2 Tomografia computadorizada da mão direita. Cortes coronal (A) e axial (B). Nota-se presença de lesão hipodensa em capitato direito, bem definida, com margens hiperdensas, sem acometimento de outros óssos da mão.

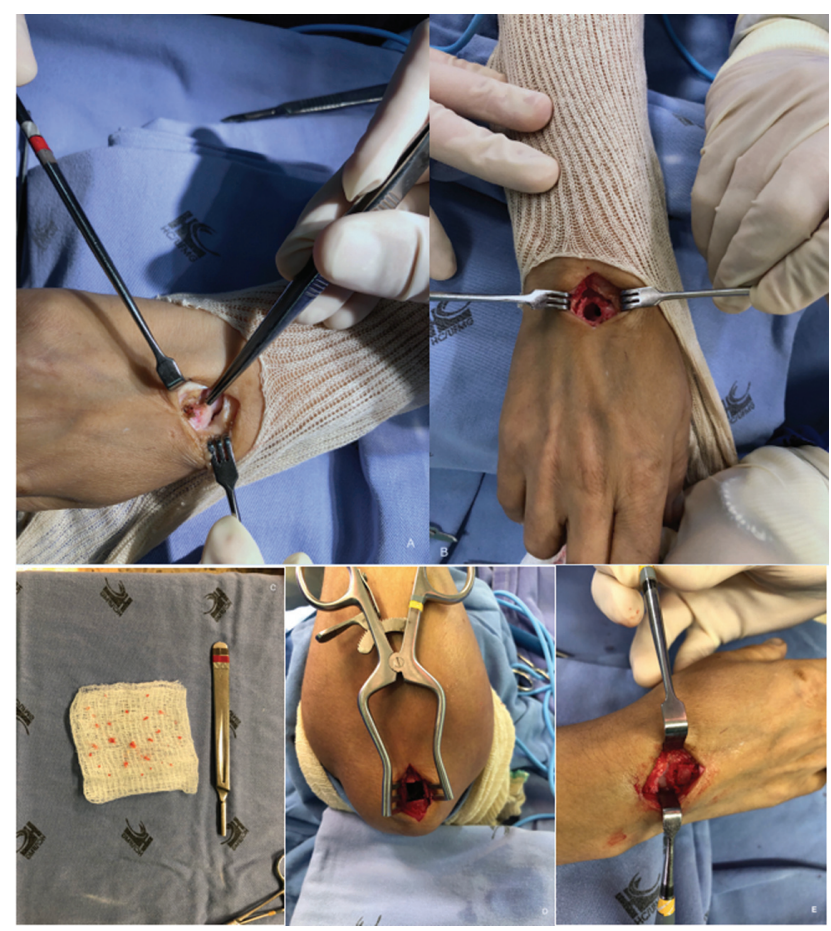

Fig. 3 Peroperatório. (A) Aspecto peroperatório da lesão em capitato direito. (B) Cavidade óssea após curetagem da lesão. (C) Material curetado enviado para exame anatomopatológico. (D) Área doadora de enxerto ósseo do olecrano direito. (E) Cavidade óssea preenchida por enxerto de olecrano. 


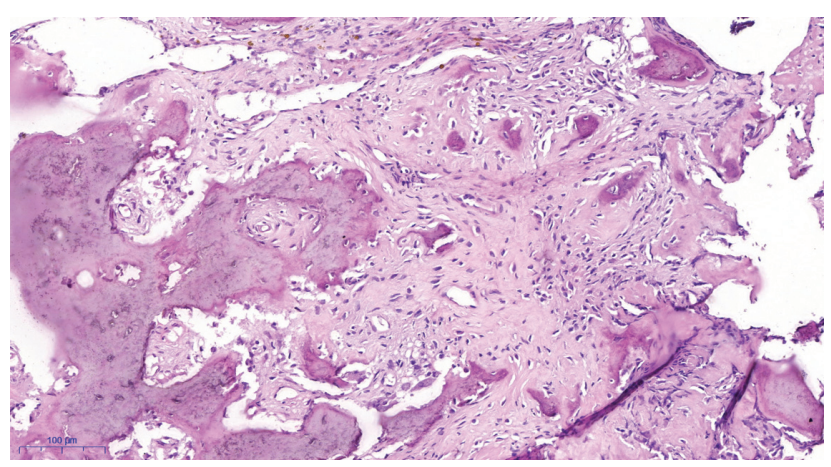

Fig. 4 Aspecto histopatológico do material enviado para análise. Observa-se tecido ósseo jovem e algumas trabéculas de tecido ósseo maduro, sem atipias, permeados por tecido fibroso.

A paciente apresentou boa recuperação funcional segundo os parâmetros do questionário Disabilities of the Arm, Shoulder and Hand (DASH, Disfunções do Braço, Ombro e Mão), e retornou gradualmente às suas atividades.

\section{Discussão}

Relatos de osteoblastoma que afeta os ossos do carpo são infrequentes, sendo o acometimento do capitato ainda mais raro. Kaptan e Atmaka ${ }^{2}$ e Afshar ${ }^{3}$ apresentaram relato dessa localização tumoral em seus respectivos trabalhos, ao passo que Murray et al., em sua série de 26.800 tumores ósseos primários, citam apenas 1 caso de osteoblastoma do capitato.

Osteoblastomas são neoplasias benignas formadoras de tecido ósseo. Microscopicamente, caracterizam-se por osso trabecular e estroma fibrovascular com produção de tecido osteoide primitivo. ${ }^{1,5}$
Eles afetam principalmente pacientes na segunda e terceira décadas de vida, preferencialmente do sexo masculino (na razão de 2-3:1)..$^{1-3}$ No caso apresentado, a paciente estava fora do padrão epidemiológico mais comum, tratando-se de uma mulher de 40 anos.

Os osteoblastomas podem acometer qualquer osso; porém, entre $40 \%$ e $50 \%$ das lesões localizam-se na coluna vertebral, preferencialmente nos elementos posteriores. ${ }^{1}$ Raramente acometem os ossos da mão. ${ }^{2,3}$ Por essas características, o diagnóstico de osteoblastoma não é frequentemente considerado em casos de tumores ósseos primários do carpo.

Radiograficamente, o osteoblastoma caracteriza-se como nicho central mineralizado com halo radiolúcido circundante e esclerose reativa, ou como massa bem definida, mista (lítica e blástica), com margens escleróticas. ${ }^{1,5}$ Tomografia computadorizada e ressonância magnética podem mostrar massa circunscrita, heterogênea, que apresenta componentes císticos, edema e osso reativo. ${ }^{5}$ Dor progressiva, constante, sem resposta a salicilatos é o sintoma mais comum, ${ }^{6}$ como observado no caso descrito.

As lesões osteolíticas não são causa frequente de dor crônica no punho, ${ }^{6}$ mas devem ser pensadas principalmente na ausência de histórico de trauma local. Alguns exemplos são o gânglio intraósseo, o osteoma osteoide, o encondroma, e o osteoblastoma. ${ }^{6,7}$ Conhecer as características clínicas de cada uma dessas lesões é fundamental para diferenciá-las.

Uma dúvida frequente é a diferenciação entre osteoblastoma e osteoma osteoide. Na mão, o osteoma osteoide é mais frequente. ${ }^{8}$ Classicamente, apresenta-se como lesão $<1,5 \mathrm{~cm}$, com nidus central circundado por zona de esclerose. A dor é intensa, pior à noite, com alívio importante com o uso de salicilatos. ${ }^{5}$

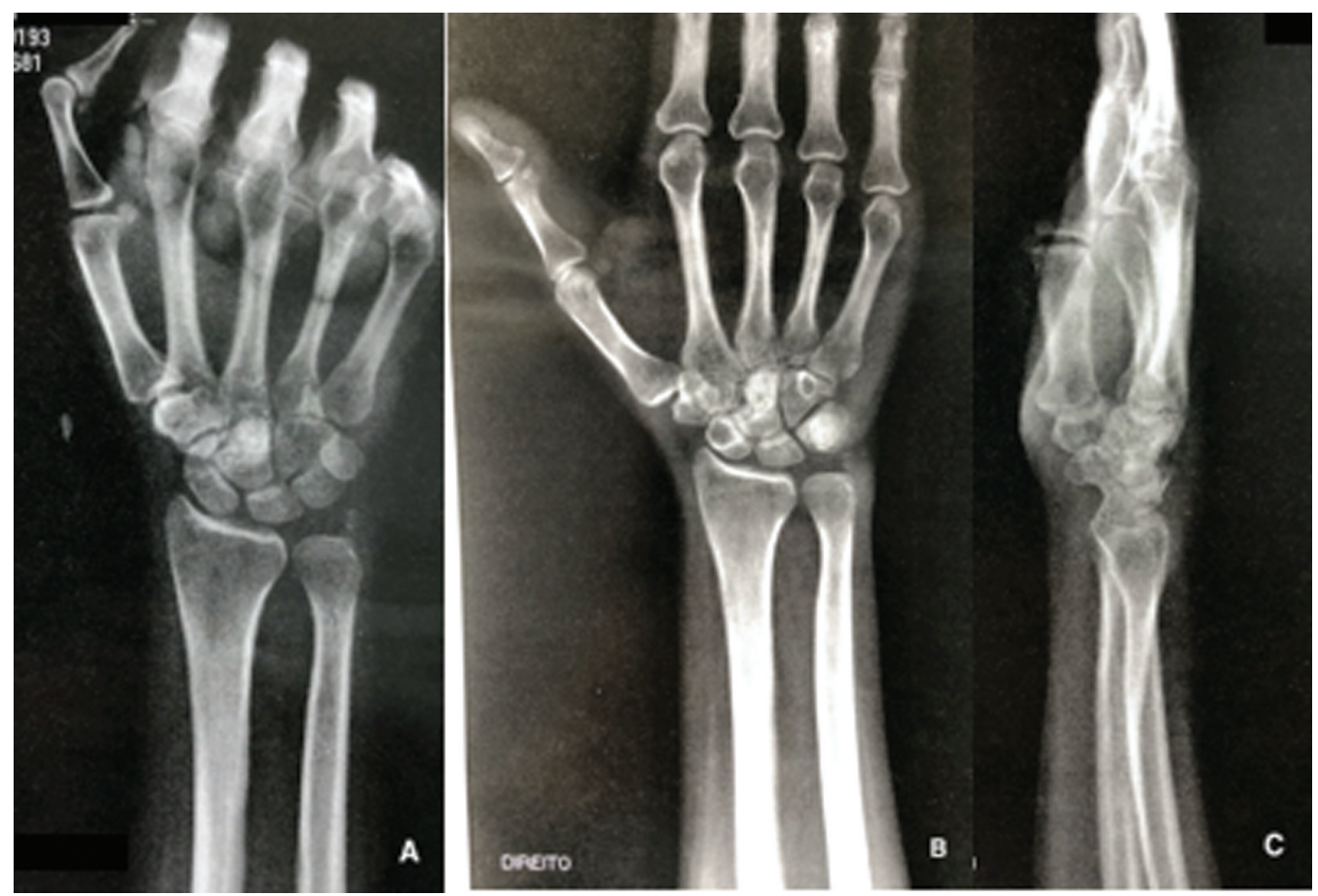

Fig. 5 Radiografias do punho direito com oito semanas de pós-operatório. Observa-se boa integração do enxerto ósseo ao capitato direito, sem sinais de recidiva da lesão. Radiografia posteroanterior com o punho cerrado (A), posteroanterior (B) e de perfil (C) do punho direito. 
Microscopicamente, as lesões são muito semelhantes, sendo o revestimento das trabéculas ósseas por uma camada única de osteoblastos, a circunscrição afilada, e o arranjo solto do tecido características que favorecem o diagnóstico de osteoblastoma. ${ }^{1}$

No caso dos tumores que envolvem os ossos da mão, o encondroma é diagnóstico diferencial importante, uma vez que se trata do tumor primário ósseo mais comum das mãos. ${ }^{6,8}$ Sua aparência radiográfica clássica é a de lesão osteolítica bem definida, com calcificações pontilhadas. ${ }^{6}$

Outro diagnóstico diferencial, o gânglio intraósseo é uma lesão cística benigna, sendo assintomática e um achado incidental em exames de imagem em aproximadamente $80 \%$ dos casos. ${ }^{9}$

Na radiografia, apresenta-se como lesão cística, unilocular, não expansiva, bem definida, com bordas escleróticas. A ressonância magnética mostra lesão de hipossinal em T1 e hipersinal nas sequências com supressão de gordura. À microscopia, o gânglio intraósseo é caracterizado por tecido mucoide, degeneração mixomatosa de tecido conjuntivo e paredes fibrosas. ${ }^{7}$

O tratamento dos osteoblastomas ainda é motivo de debates. Estudos sugerem que o tumor pode sofrer transformação maligna, ${ }^{7}$ além de taxas de recorrência entre $10 \%$ e $20 \%{ }^{3,5}$ Sendo assim, há autores que indicam desde ressecção completa da lesão até curetagem associada ou não a enxertia óssea. ${ }^{3,10}$ Castelló et al. ${ }^{10}$ descreveram bons resultados após curetagem seguida de preenchimento com enxerto ósseo.

No caso relatado, inicialmente, o quadro clínico e a aparência radiográfica não permitiram um diagnóstico específico da lesão osteolítica. Contudo, em função do comportamento não agressivo da lesão, suas características benignas, e a ausência de extensão para partes moles ou outros ossos aos exames de imagem, optou-se pela realização de curetagem com preenchimento com enxerto ósseo, sendo o material coletado enviado para exame anatomopatológico, que sugeriu posteriormente o diagnóstico de osteoblastoma.

Apresentou-se um caso raro de osteoblastoma do capitato como causa de dor crônica do punho, assim como foi feita uma revisão e atualização do tema. Dessa forma, o osteoblastoma pode ser considerado um diagnóstico de exclusão quando nos deparamos com uma lesão osteolítica no nível do carpo.

\section{Conflito de Interesses}

Os autores declaram não haver conflito de interesses.

\section{Referências}

1 Canale ST, Beaty JH. Campbell Cirurgia Ortopédica. 12 ed. Rio de Janeiro: Elsevier; 2017

2 Kaptan C, Atmaca H. Osteoblastoma of the os capitatum. Case Rep Orthop 2014;2014:241716

3 Afshar A. Osteoblastoma of the capitate bone. J Hand Microsurg 2012;4(01):34-38

4 Murray PM, Berger RA, Inwards CY. Primary neoplasms of the carpal bones. J Hand Surg Am 1999;24(05):1008-1013

5 Zhang Y, Rosenberg AE. Bone-Forming Tumors. Surg Pathol Clin 2017;10(03):513-535

6 Lubahn JD, Bachoura A. Enchondroma of the Hand: Evaluation and Management. J Am Acad Orthop Surg 2016;24(09):625-633

7 Salunke AA, Singh S, Kanani H, et al. Intraosseous Ganglion Cyst of Scaphoid: An Uncommon Cause of Radial Wrist Pain. J Hand Surg Asian Pac Vol 2016;21(01):109-112

8 Simon MJ, Pogoda P, Hövelborn F, et al. Incidence, histopathologic analysis and distribution of tumours of the hand. BMC Musculoskelet Disord 2014;15:182

9 Osagie L, Gallivan S, Wickham N, Umarji S. Intraosseous ganglion cysts of the carpus: current practice. Hand (N Y) 2015;10(04): 598-601

10 Castelló JR, Garro L, San Miguel P, Campo M. Osteoblastoma of the scaphoid-long-term results following curettage: a case report. J Hand Surg Am 1996;21(03):426-427 